OPEN ACCESS

Edited by:

Fabrice Merien,

Auckland University of Technology,

New Zealand

Reviewed by:

Haider Abdul-Lateef Mousa,

University of Basrah, Iraq

Gabriele Margos,

Ludwig-Maximilians-Universität

München, Germany

*Correspondence:

Yee L. Lau

lauyeeling@um.edu.my

Specialty section

This article was submitted to

Infectious Diseases,

a section of the journal

Frontiers in Microbiology

Received: 12 January 2016

Accepted: 12 April 2016

Published: 27 April 2016

Citation:

Ching XT, Fong MY and Lau YL (2016)

Evaluation of Immunoprotection

Conferred by the Subunit Vaccines of GRA2 and GRA5 against Acute

Toxoplasmosis in BALB/C Mice.

Front. Microbiol. 7:609.

doi: 10.3389/fmicb.2016.00609

\section{Evaluation of Immunoprotection Conferred by the Subunit Vaccines of GRA2 and GRA5 against Acute Toxoplasmosis in BALB/c Mice}

\author{
Xiao T. Ching, Mun Y. Fong and Yee L. Lau* \\ Department of Parasitology, Faculty of Medicine, University of Malaya, Kuala Lumpur, Malaysia
}

Toxoplasmosis is a foodborne disease caused by Toxoplasma gondii, an obligate intracellular parasite. Severe symptoms occur in the immunocompromised patients and pregnant women leading to fatality and abortions respectively. Vaccination development is essential to control the disease. The T. gondii dense granule antigen 2 and 5 (GRA2 and GRA5) have been targeted in this study because these proteins are essential to the development of parasitophorous vacuole (PV), a specialized compartment formed within the infected host cell. PV is resistance to host cell endosomes and lysosomes thereby protecting the invaded parasite. Recombinant dense granular proteins, GRA2 (rGRA2) and GRA5 (rGRA5) were cloned, expressed, and purified in Escherichia coli, BL21 (DE3) pLysS. The potential of these purified antigens as subunit vaccine candidates against toxoplasmosis were evaluated through subcutaneous injection of BALB/c mice followed by immunological characterization (humoral- and cellular-mediated) and lethal challenge against virulent T. gondii RH strain in BALB/c mice. Results obtained demonstrated that rGRA2 and rGRA5 elicited humoral and cellular-mediated immunity in the mice. High level of $\operatorname{lgG}$ antibody was produced with the isotype $\operatorname{lgG} 2 \mathrm{a} / \mathrm{lgG} 1$ ratio of $\approx 0.87$ $(p<0.001)$. Significant increase $(p<0.05)$ in the level of four cytokines (IFN- $\gamma$, IL2, IL-4, and IL-10) was obtained. The antibody and cytokine results suggest that a mix mode of Th1/Th2-immunity was elicited with predominant Th1-immune response inducing partial protection against T. gondii acute infection in BALB/c mice. Our findings indicated that both GRA2 and GRA5 are potential candidates for vaccine development against $T$. gondii acute infection.

Keywords: Toxoplasma gondii, toxoplasmosis, GRA2, GRA5, subunit vaccine

\section{INTRODUCTION}

Toxoplasma gondii (T. gondii) is a ubiquitous and obligate intracellular protozoan parasite capable of infecting a broad range of warm-blooded hosts (Dubey, 2010), causing a disease known as toxoplasmosis. Toxoplasmosis is a common infection globally distributed affecting up to onethird of the world's human population (Jackson and Hutchison, 1989). It poses danger to the AIDS patients and pregnant women where fatality and abortions can result respectively. T. gondii infection also causes abortions in livestock especially sheep and goats, leading to great economic losses in livestock and food industry (Buxton, 1998). 
Infected patients are commonly treated with pyrimethamine, sulphadiazine and spiramycin (during pregnancy) but these drugs are unable to eliminate the parasites completely (Hill and Dubey, 2002; Montoya and Liesenfeld, 2004). The problem of parasites eradication, disease reactivation, toxic effects and emerging drug resistance in parasites makes drug treatment unreliable for long term treatment (Bhopale, 2003; Kur et al., 2009; Innes, 2010). Development of effective vaccines against toxoplasmosis is thus needed to fight against the parasite. To date, Toxovax is the only available vaccine in the market for preventing toxoplasmosis in domestic animals especially sheep and goats. However, this vaccine is not widely acceptable for human use due to the high possibility of regaining the parasite's pathogenicity (Chen et al., 2009), side effects and high cost of production (Ismael et al., 2003). Production of safe recombinant vaccines is made possible through recombinant DNA technology.

Development of protein-based vaccines are basically safer and more specific in boosting the immune response of the recipients by presenting only selected immunogenic antigens instead of the whole parasite (Schaap et al., 2007). The common route of purified recombinant protein injection is via subcutaneous tissue. Upon injection, the proteins will be taken up by circulating antigen presenting cell (APC) such as macrophage. The proteins will then be processed into peptide-MHC class II complex within APC before being presented on the cell surface to $\mathrm{CD} 4^{+}$ helper $\mathrm{T}$ cells, stimulating humoral-mediated immunity (Th2) resulting in antibody production. Difficulties in generating Th1 immunity can be overcome by formulating the recombinant proteins with appropriate adjuvants as they play important role in directing the desired Th1/Th2 profiles (Kur et al., 2009; Bruna-Romero et al., 2012). For example, formulation of alum (Th2 inducer) and IL-12 (Th1 inducer) result in a strong Th1 activity (Schaap et al., 2007). Other adjuvants that are commonly used in subcutaneous injection are Freund's complete adjuvant (FCA), Freund's incomplete adjuvant (FIA), liposomes and IL-12.

Toxoplasma gondii infection begins when the tachyzoites invade host cells. Uncontrolled replication of the tachyzoites leads to rupturing of the infected cells thereby releasing new parasites to invade neighboring cells. The parasite remains protected within a parasitophorous vacuole $(\mathrm{PV})$, a specialized compartment formed within the infected host cell during and after invasion. Dense granules (GRAs) are T. gondii specialized secretory organelles involved in PV development whereby the antigens helped in the maturation and modification of both PV and PV membrane (Nam, 2009). GRAs are the major components of both vacuole surrounding tachyzoites and encysted bradyzoites (Capron and Dessaint, 1988; CesbronDelauw and Capron, 1993) which have been identified as potential vaccines (Scorza et al., 2003; Hiszczynska-Sawicka et al., 2011; Sun et al., 2011).

GRA2 contributes to the formation of intravacuolar network in $\mathrm{PV}$, allowing proteins and nutrients transportation to nourish the parasites while GRA5 helps to inhibit apoptosis of the infected cells thereby protecting the parasites during cell invasion (Feng et al., 2002; Nam, 2009). Both GRA2 and GRA5 are expressed throughout the whole intermediate host life cycle of $T$. gondii thus preventing stage-limited protection against toxoplasmosis (Tilley et al., 1997; Zhou et al., 2007).

Several studies had been conducted on the evaluation of multi-component vaccine candidate incorporating GRA2 or GRA5 with other potential genes against toxoplasmosis (Zhou et al., 2007; Igarashi et al., 2008a; Xue et al., 2008; Liu et al., 2009). However, limited number of study had been performed on these two target genes as single antigen vaccine especially GRA5. The only report on rGRA2 expressed in Escherichia coli as single subunit vaccine candidate investigated its efficacy against chronic toxoplasmosis based on the T. gondii brain cysts counts (Golkar et al., 2007). Nevertheless, protective effect conferred by the same antigen against lethal parasitic infection of type I virulent strain has not been reported yet. In this study, recombinant GRA2 and GRA5 proteins were subjected to mice immunization study as single antigen subunit vaccine candidates against acute T. gondii infection in $\mathrm{BALB} / \mathrm{c}$ mice.

\section{MATERIALS AND METHODS}

\section{Mice}

Six- to eight-week old female BALB/c mice were purchased from Monash University Sunway Campus. The mice were maintained in a pathogen free environment and were fed ad lib with commercial food pellets and water.

\section{Ethics Statement}

This study was carried out in strict accordance with the recommendations in the Guide for the Care and Use of Laboratory Animals of the National Institutes of Health. The protocol was approved by the Institutional Animal Care and Use Committee (IACUC) of the University of Malaya, Faculty of Medicine (Permit Number: 2014-06-03/PARA/R/CXT).

\section{Parasites Propagation and Harvest}

Toxoplasma gondii tachyzoites of the virulent wild-type RH strain were provided by the Department of Parasitology, University of Malaya, Kuala Lumpur, Malaysia. They were propagated in vitro involving infection of Human Foreskin Fibroblast (HFF) cells. DMEM complete medium was replaced with parasite infection medium 12-16 h pre-infection when the growth of HFF cells reached $80-90 \%$ confluence. Freshly isolated or frozen parasites were washed with sterile phosphate-buffered saline (PBS) and resuspended in parasite infection medium before infecting the HFF cells. After $24 \mathrm{~h}$ of incubation in the $\mathrm{CO}_{2}$ incubator, the infected cells were replaced with new parasite infection medium in order to remove free floating tachyzoites. Incubation was continued until the lysis of the infected cells triggered by the actively replicating tachyzoites thereby releasing them into the medium.

During lysis of the infected HFF cells, erupted tachyzoites and the infected cells were harvested with a cell scraper (TPP, USA). The entire cell suspension was transferred to a syringe attached to a 25 gage needle which was placed into a $50 \mathrm{ml}$ polypropylene tube beforehand. The cell suspension was forced to pass through the needle by the plunger to ensure the release of tachyzoites from 
the intact infected cells. The act was repeated twice followed by two times of washing with sterile PBS whereby the cell suspension was centrifuged at $1,000 \times g$ for 15 min between each wash. After the last wash, the pellet obtained was resuspended in sterile PBS before filtering through $3 \mu \mathrm{m}$ polycarbonate membrane (Merck, USA). The filtrate containing only the parasites was sedimented and resuspended in sterile PBS prior to usage.

\section{Recombinant GRA2 and GRA5 Proteins Production}

Recombinant GRA2 and GRA5 plasmids were constructed and the sequences were verified. Heterologous expression of the positive clones was performed in BL21 (DE3) pLysS followed by affinity purification. Identity of the purified recombinant proteins were validated through MALDI-TOF MS analysis before subjected to vaccination study. The entire procedures were described thoroughly in our previous report (Ching et al., 2015).

\section{Immunization Regime}

Six- to eight-week old female inbred BALB/c mice were divided into four immunization groups with 13 mice in each group; two negative controls: PBS and pRSET B, two protein groups: rGRA2 and rGRA5. Three injections were administered subcutaneously with final protein dose of $10 \mu \mathrm{g}$ at 2 weeks intervals. Injection samples were emulsified with complete/incomplete Freud's adjuvant (C/IFA) at 1:1 ratio before immunizing the mice. Blood $(50-100 \mu \mathrm{l})$ were collected from the immunized mice through tail-bleeding on day $0,14,28$, and 42 .

\section{Evaluation of Humoral Response}

Mice serum samples harvested were analyzed by western blot assay (Ching et al., 2015) and in-house ELISA against the purified recombinant proteins to detect the presence of antigen-specific IgG antibodies and to determine antibody titers.

\section{IgG Titer and Subclass Determination}

The 96-well flat bottom microplate was coated overnight at $4^{\circ} \mathrm{C}$ with $10 \mu \mathrm{g} / \mathrm{ml}$ rGRA2 or rGRA5 diluted in $100 \mu \mathrm{l}$ coating buffer. The antigen solutions were aspirated and the wells were washed three times with $0.05 \%$ PBS-T after overnight incubation. The subsequent incubation steps were all carried out at $37^{\circ} \mathrm{C}$. Non-specific binding sites of the wells were blocked by incubation with $200 \mu \mathrm{l}$ of $10 \%$ blocking buffer for $1 \mathrm{~h}$. The blocking buffer was then aspirated and the plate was washed thrice followed by incubation with $100 \mu \mathrm{l}$ of serially diluted mice sera (control and vaccinated) for another $1 \mathrm{~h}$ to determine the optimal working dilution. The plate was washed again in the same way. Bound antigen-specific IgG was detected through incubation with $100 \mu \mathrm{l}$ of diluted HRP-conjugated goat anti-mouse IgG (1:2000) for $1 \mathrm{~h}$. The plate was washed five times and the enzymatic reaction was developed by addition of $100 \mu 13,3^{\prime}, 5,5^{\prime}$-Tetramethylbenzidine (TMB), a chromogenic substrate and was incubated for 10$15 \mathrm{~min}$ at RT. The reaction was eventually stopped with $2 \mathrm{M}$ of sulphuric acid and the absorbance was measured at $450 \mathrm{~nm}$ with microplate reader. Primary and secondary antibodies were diluted in $10 \%$ blocking buffer. All samples were run in triplicates. Vaccinated mice sera were considered positive if the mean optical density (OD) of triplicate determinations was greater than the cut-off limit of the negative control groups; cut-off $=$ mean $\mathrm{OD}+2$ (standard deviation). The entire steps were repeated for IgG subclass determination assay whereby different secondary antibodies involved were HRP-conjugated goat anti-mouse IgG1 and IgG2a.

\section{In Vitro Splenocyte Proliferation Assay}

Three mice per group were euthanized with $\mathrm{CO}_{2}$ and spleens were harvested aseptically two weeks after final immunization. Single cell suspension was prepared by mashing the spleen over a $70 \mu \mathrm{m}$ cell strainer and washed with $10 \mathrm{ml}$ RPMI complete medium (CM) before subjected to centrifugation. All centrifugation steps were performed at $1,500 \mathrm{rpm}$ for $10 \mathrm{~min}$. Cell pellet obtained was resuspended with $5 \mathrm{ml}$ of ACK lysis buffer and incubated for $5 \mathrm{~min}$ at RT. Five minute later; the cell suspension was washed with $20 \mathrm{ml} \mathrm{CM}$ and was centrifuged again. This step was repeated with $10 \mathrm{ml}$ CM. Cell pellet obtained was eventually resuspended in $10 \mathrm{ml} \mathrm{CM}$. Splenocytes were cultured in 96-well flat bottom microplate with cell density of $2 \times 10^{5}$ cells/well in triplicates. The cells were induced with culture medium alone (negative control), $10 \mu \mathrm{g} / \mathrm{ml} \mathrm{rGRA} 2$ or rGRA 5 or $5 \mu \mathrm{g} / \mathrm{ml}$ con A (positive control) before incubated at $37^{\circ} \mathrm{C}$ in a $5 \% \mathrm{CO}_{2}$ incubator for 24,72 , and $96 \mathrm{~h}$.

Splenocytes proliferation was analyzed with MTT Cell Proliferation Kit at $72 \mathrm{~h}$ post-incubation according to the instruction's manual. Briefly, MTT labeling reagents was added into each well of cells and were incubated for $4 \mathrm{~h}$ followed by incubation overnight with $100 \mu \mathrm{l}$ of Solubilization solution. The plate was read at $570 \mathrm{~nm}$ with microplate reader the next day.

Stimulation index (S1)

$$
=\frac{\text { mean } \mathrm{OD}_{570} \text { values of stimulated cells }}{\text { mean } \mathrm{OD}_{570} \text { values of unstimulated cells }}
$$

\section{Cytokine Assays}

Splenocytes cultured and incubated at different time point (24, 72 , and $96 \mathrm{~h}$ ) were subjected to centrifugation at 2,000 $\times g$ for $20 \mathrm{~min}$. Culture supernatants were collected for various cytokine assays such as IFN- $\gamma$, IL-2, IL-4 and IL-10 assays. These assays were performed in accordance with the instruction's manuals.

\section{Mice Challenge}

The remaining vaccinated and control mice were subjected to lethal parasitic challenge study through intraperitoneal injection of $1 \times 10^{3}$ live tachyzoites of $T$. gondii virulent $\mathrm{RH}$ strain. Mortality rate of the mice were monitored and recorded twice daily whereby the infected mice were observed for endpoint criteria that is when they were heavily infected, showing symptoms of sluggish movement, hunched back posture, ruffled and thinning hair coat as well as obvious reduced food and 


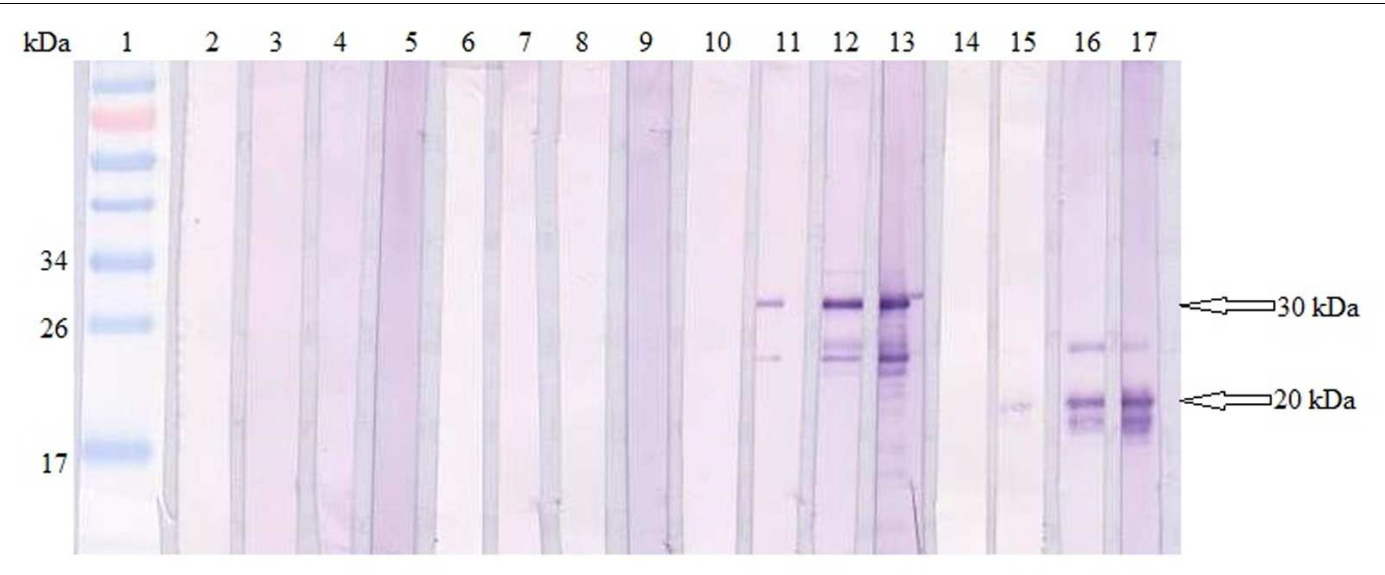

FIGURE 1 | Qualitative detections of total specific anti-GRAs IgG antibodies in mice sera. Western blots of purified recombinant proteins (rGRA2 or/and rGRA5) with sera of the immunized mice. Lane 1 contained PageRuler Prestained Protein Ladder. Lane 2-5 were incubated with sera of PBS-injected mice, lane 6-9 were incubated with sera of pRSET B-injected mice, lane 10-13 were incubated with sera of rGRA2-immunized mice and lane 14-17 were incubated with sera of rGRA5-immunized mice. The 4 sera represented sera collected at week 0, 2, 4, and 6 post-prime injections. The $30 \mathrm{kDa}$ purified rGRA2 and $20 \mathrm{kDa}$ purified rGRA5 were first detected at week 2 (lane 11 and 15 respectively) followed by an increase in the band intensity at week 4 and 6 . No bands were observed in the mice sera of the two control groups.

water consumption. The heavily infected mice that reached endpoint criteria were humanely killed by exposure to a gradually increasing concentration of carbon dioxide $\left(\mathrm{CO}_{2}\right)$ inside a closed chamber.

\section{Statistical Analysis}

Significance levels of the differences between groups of mice were analyzed through Student's $t$-test or analysis of variance (ANOVA). $P<0.05$ indicates statistical significance. The survival rate was calculated based on $\chi^{2}$ (chi-square) test while the survival graph was drawn based on Kaplan-Meier method (Kaplan and Meier, 1958).

\section{RESULTS}

\section{IgG Antibody Detection}

Total specific anti-GRA2 and anti-GRA5 IgG antibodies were detected in the sera collected from the mice immunized with rGRA2 and rGRA5 respectively through SDS-PAGE/WB (Figure 1) and ELISA (Figure 2; Table 1) against purified recombinant proteins. Faint protein bands with target size of 30 and $20 \mathrm{kDa}$ were observed at week 2 after prime injection of mice with rGRA2 and rGRA5 respectively as shown in Figure 1. The intensity increased at week 4 and 6 following first and second booster injections. However, antibody detection was not observed in Native-PAGE/WB (data not shown). Meanwhile, Figure 2 also showed the same phenomena whereby significantly higher levels of IgG antibodies were observed in the recombinant protein-vaccinated groups compared to two control groups $(p<0.001)$ and the level gradually elevated with successive immunizations. There was no statistical difference between the two control groups at week 2 and $4(p>0.05)$. However, IgG level in pRSET B-injected group was found significantly

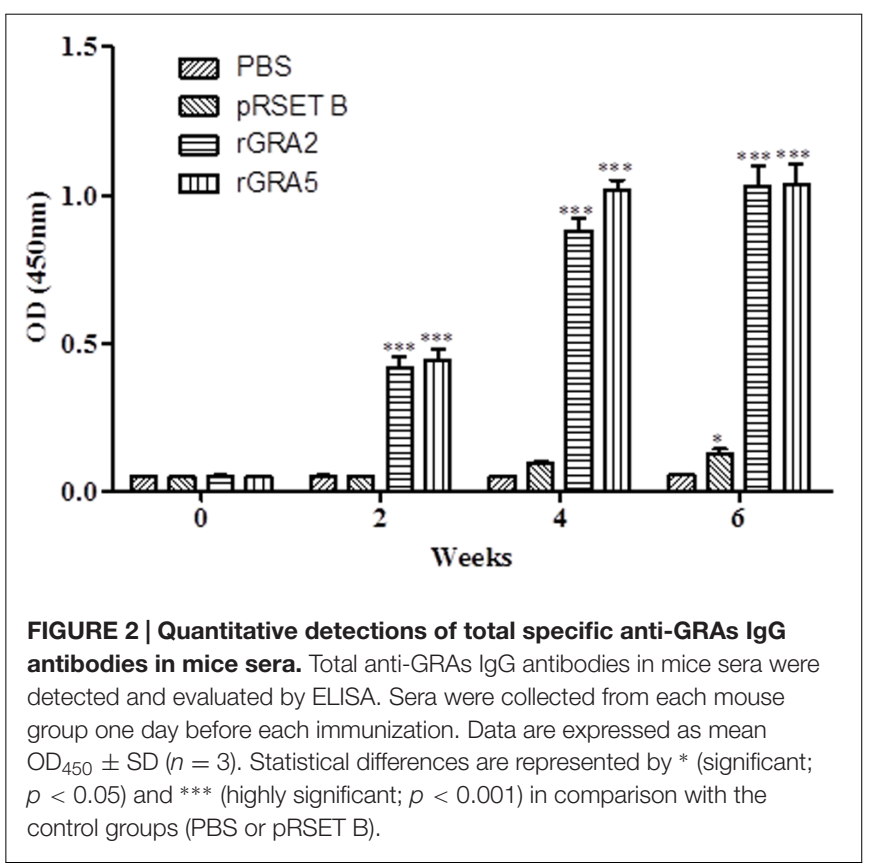

higher than that of PBS-injected group $(p<0.05)$ at week 6 post-prime injection. The level of IgG antibodies in mice sera collected from rGRA5-vaccinated group was significantly higher than that of rGRA2-vaccinated group $(p<0.001)$ at week 4 but both groups reached highest level 2 weeks after the last injection (week 6) without statistical difference $(p>0.05)$. These results indicated that both recombinant GRA2 and GRA5 proteins are immunogenic and capable of stimulating significantly strong humoral immune response in the respective vaccinated mice compared to the negative control groups. 
TABLE 1 | Specific anti-GRAs IgG antibody profile in sera collected from the immunized BALB/c mice 2 weeks after last injection.

\begin{tabular}{lccc}
\hline Group $(\boldsymbol{n}=\mathbf{3})$ & \multicolumn{3}{c}{ OD $_{\mathbf{4 5 0}}$} \\
\cline { 2 - 4 } & \multicolumn{1}{c}{ IgG } & IgG1 & IgG2a \\
\hline rGRA2 & $1.0330 \pm 0.0645^{* * *}$ & $2.9760 \pm 0.2025^{* * *}$ & $2.5850 \pm 0.0303^{* * *}$ \\
rGRA5 & $1.0370 \pm 0.0679^{* * *}$ & $3.0770 \pm 0.0528^{* * *}$ & $2.6820 \pm 0.0768^{* * *}$ \\
pRSET B & $0.1267 \pm 0.0170^{*}$ & $0.0801 \pm 0.0020$ & $0.0686 \pm 0.0033$ \\
PBS & $0.0540 \pm 0.0007$ & $0.1256 \pm 0.0060$ & $0.0717 \pm 0.0078$ \\
\hline
\end{tabular}

Data are expressed as mean $\pm S D$. Statistical differences are represented by * (significant; $p<0.05$ ) and ${ }^{* * *}$ (highly significant; $p<0.001$ ) in comparison with the control groups (PBS or pRSET B). Each group consisted of three mice.

\section{IgG Titer Determination}

Antibody titer is a quantitative measurement of the amount of antibody capable of recognizing the respective epitope. It is usually expressed as reciprocal of the highest dilution with an OD450 greater than the positive cut-off value of IgG (mean $+2 \mathrm{SD}$ ) relative to the control mice sera at the same dilution. Antibody titer of both the anti-GRA2 and anti-GRA5 IgG was determined to range from $1: 409,600$ to $1: 819,200$ by ELISA.

\section{IgG Antibody Isotypes Determination}

Polyclonal antibody isotypes (IgG1 and IgG2a) in the immunized $\mathrm{BALB} / \mathrm{c}$ mice sera were further assessed by ELISA in order to identify type of immunity being triggered. The levels of specific anti-GRAs IgG1 and IgG2a being produced are depicted in Figure 3 and tabulated in Table 1. Generally, the level of IgG isotypes present in the sera of the two vaccinated mice groups were highly significantly greater than that of the two control groups $(p<0.001)$. No statistical difference was observed between the two control groups $(p>0.05)$ and also between the two vaccinated groups $(p>0.05)$. On top of that, it was

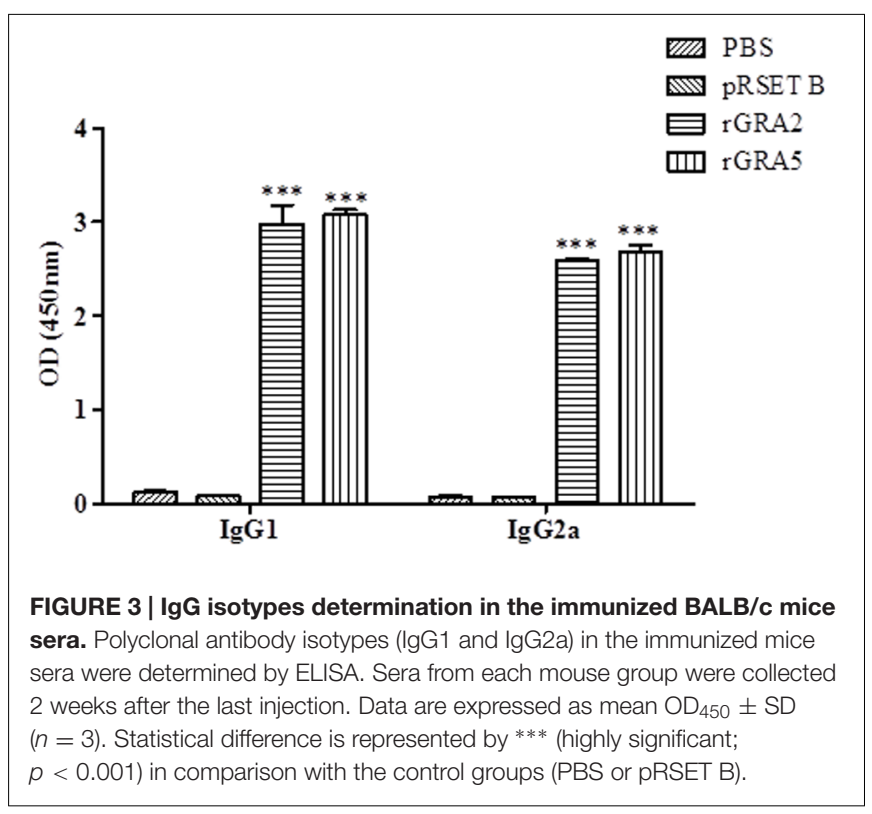

shown that high levels of two IgG isotypes were detected in all the rGRA2- and rGRA5-immunized mice sera, with slightly higher level of IgG1 compared to IgG2a, giving rise to an IgG2a/IgG1 ratio of $<1(\approx 0.87)$. The result obtained indicated that both Th1 and Th2 immune responses were driven in all the vaccinated mice.

\section{In Vitro Splenocytes Proliferation Assay}

Antigen-specific proliferative response of splenocytes from each mice group to rGRA2 or/and rGRA5 stimulus was determined using MTT assay and represented by the SI value as illustrated in Figure 4 and Table 2. Generally, significantly higher SI value was observed in the recombinant protein-vaccinated groups compared to the control groups $(p<0.05)$. On top of that, splenocytes from rGRA5-vaccinated mice had significantly stronger proliferation compared to that of rGRA2-vaccinated mice in response to their respective stimulus $(p<0.05)$. Nevertheless, there was no statistical difference between the two control groups $(p>0.05)$. Meanwhile, SI value for all mice groups had comparable levels in response to the mitogen conA. These results indicated that $\mathrm{T}$ lymphocytes of the vaccinated mice were successfully stimulated.

\section{Cytokine Production Assay}

Results obtained showed that splenocytes of the vaccinated mice produced significantly higher level of IFN- $\gamma$ and IL- 2 compared to the control groups $(p<0.05)$ as demonstrated in Figure 5 and Table 2. No statistical difference was observed between two vaccinated groups $(p>0.05)$ and between two control groups $(p>0.05)$. In contrast, relatively low levels of IL- 4 and IL-10 were released by the stimulated splenocytes of the mice immunized with rGRA2 and rGRA5 (Figure 6; Table 2). Undetectable level of

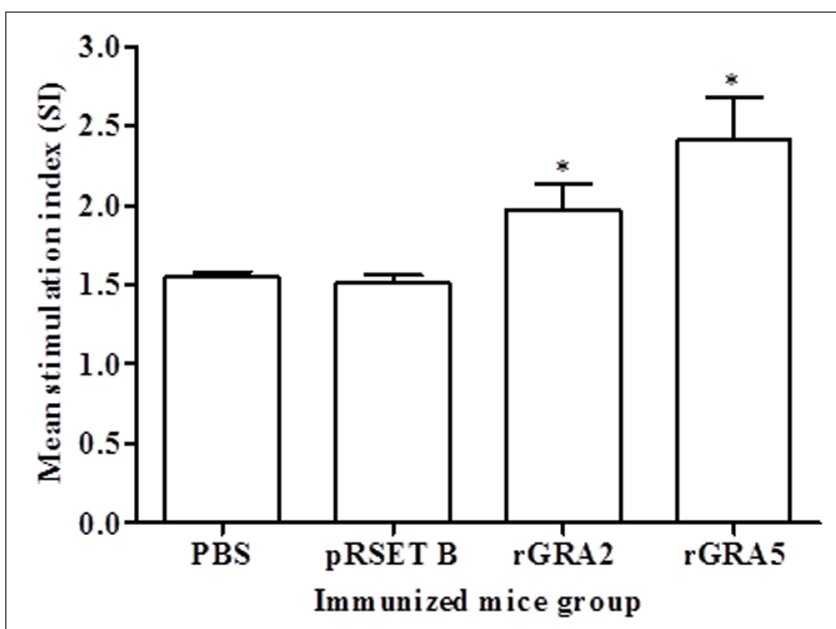

FIGURE 4 | In vitro splenocytes proliferation response in mice. Spleen lymphocytes were harvested from mice immunized with rGRA2, rGRA5, pRSET B and PBS 2 weeks after last injection. The splenocytes were cultured and stimulated with the respective recombinant proteins. Proliferative response was measured by MTT assay. Data are expressed as mean stimulation index $(\mathrm{SI}) \pm \mathrm{SD}(n=3)$. Statistical difference is represented by $*(p<0.05)$ in comparison with the control groups (PBS or pRSET B). 
TABLE 2 | Characterization of cellular-mediated immunity in the vaccinated mice.

\begin{tabular}{|c|c|c|c|c|c|}
\hline \multirow[t]{2}{*}{ Group $(n=3)$} & \multirow[t]{2}{*}{ Proliferation (SI) } & \multicolumn{4}{|c|}{ Cytokine level (pg/ml) } \\
\hline & & IFN- $\gamma$ & IL-2 & IL-4 & IL-10 \\
\hline rGRA2 & $1.973 \pm 0.1589 *$ & $4645 \pm 1032^{*}$ & $1527 \pm 247^{*}$ & $9.989 \pm 2.231$ & $121.6 \pm 43.94^{*}$ \\
\hline rGRA5 & $2.414 \pm 0.2674^{*}$ & $4724 \pm 372.5^{*}$ & $1232 \pm 95.07^{*}$ & $25.46 \pm 11.98^{*}$ & $178.3 \pm 27.71^{*}$ \\
\hline pRSET B & $1.514 \pm 0.0447$ & $197.4 \pm 61.45$ & $146 \pm 42.01$ & Undetectable & $61.55 \pm 16.18$ \\
\hline PBS & $1.549 \pm 0.0345$ & $142.7 \pm 77.15$ & $123 \pm 13.67$ & Undetectable & $26.98 \pm 8.667$ \\
\hline
\end{tabular}

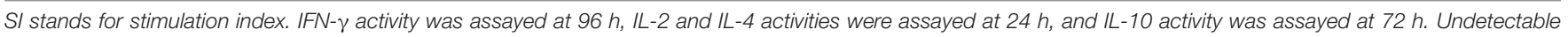

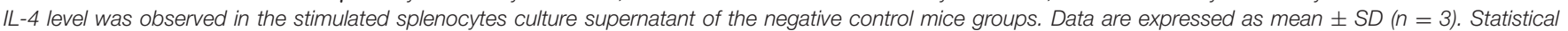
difference is represented by* $(p<0.05)$ in comparison with the control groups (PBS or pRSET B).

IL-4 was observed in the control groups (Table 2). However, IL10 level produced by splenocytes from the recombinant proteinvaccinated mice was significantly higher compared to PBS and pRSET B-injected mice $(p<0.05)$. Production of huge amount of IFN- $\gamma$ and IL-2 and relatively low level of IL-4 and IL10 indicated that Th1 immune response was favored in the vaccinated mice.

\section{Protective Efficacy of Recombinant Protein Vaccination in BALB/c Mice}

Protective efficacy of recombinant GRA2 and GRA5 proteins in the immunized BALB/c mice were evaluated against lethal challenge with $T$. gondii. The survival rates of the four challenged mice groups were illustrated in Figure 7. It was shown that the two vaccinated mice groups had significantly prolonged survival rates as compared to the two control mice groups (PBS and pRSET B) $(p<0.05)$. All PBS- and pRSET B-injected mice succumbed to the parasite infection on day 6 (median survival of 6 days) and day 9 (median survival of 8 days) respectively. Meanwhile, rGRA2- and rGRA5immunized mice died within 8-18 days post-infection with the median survival of 16.5 and 16 days respectively. Although all the immunized mice died on day 18, but these two subunit vaccines were successfully demonstrated to increase the survival rates of the vaccinated $\mathrm{BALB} / \mathrm{c}$ mice against $T$. gondii infection.

\section{DISCUSSION}

Subunit vaccination is generally known for its efficacy in inducing humoral immune responses against extracellular pathogens through antibody generation which is favored by $\mathrm{T}$ helper 2 (Th2) related responses. Th1 and Th2 responses are characterized by differences in cytokine production and by antibody isotype (Snapper and Paul, 1987; Gazzinelli et al., 1991; Germann et al., 1995; Bessieres et al., 1997). However, T. gondii is an intracellular parasite, thus an immune response mediated by $\mathrm{CD} 4^{+} \mathrm{Th} 1$ and $\mathrm{CD}^{+}$cytotoxic $\mathrm{T}$ cells are the main components required to combat this parasitic infection (Denkers and Gazzinelli, 1998). Immunization using animal model with recombinant expressed protein alone is weakly immunogenic (Sloat et al., 2010) and often elicited a mixed Th1/Th2-like response with higher tendency of IgG1 isotype production, driving predominantly Th2-like response (Echeverria et al., 2006; Dziadek et al., 2009, 2012; Sun et al., 2014). Therefore, addition of Th1-directing adjuvant such as Complete Freund's adjuvant (CFA) was used in this study. This

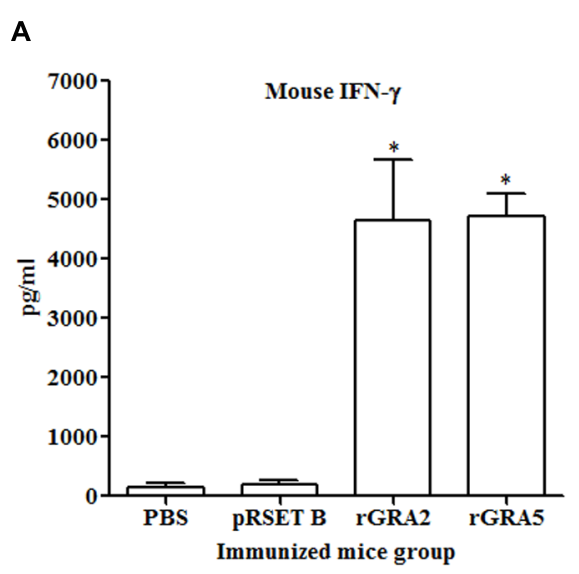

B

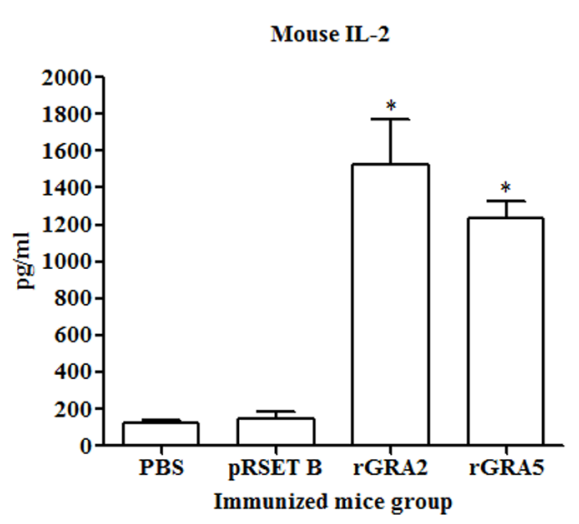

FIGURE 5 | (A,B) IFN- $\gamma$ and IL-2 production by the stimulated splenocytes of the immunized mice. Culture supernatants from the antigen-stimulated immunized mice splenocytes were harvested at 96 and $24 \mathrm{~h}$ post-incubation for the evaluation of (A) IFN- $\gamma$ and (B) IL-2 production respectively via ELISA. Data are expressed as mean $\pm \mathrm{SD}(n=3)$. Statistical difference is represented by * $(p<0.05)$ in comparison with the control groups (PBS or pRSET B). 
A

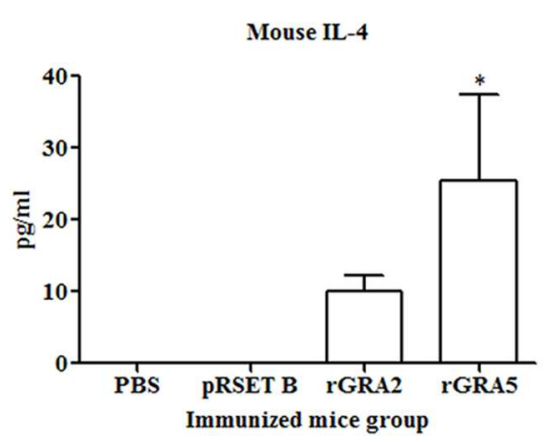

B

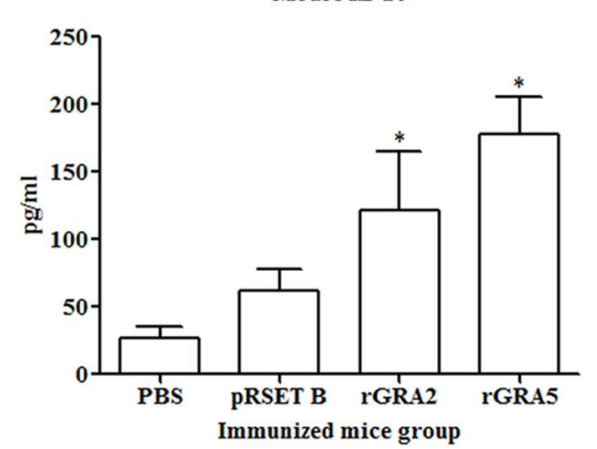

FIGURE 6 | (A,B) IL-4 and IL-10 production by the stimulated splenocytes of the immunized mice. Culture supernatants from the antigen-stimulated immunized mice splenocytes were harvested at 24 and $72 \mathrm{~h}$ post-incubation for the evaluation of (A) IL-4 and (B) IL-10 production respectively via ELISA. Data are expressed as mean $\pm \mathrm{SD}(n=3)$. Statistical difference is represented by * $(p<0.05)$ in comparison with the control groups (PBS or pRSET B).

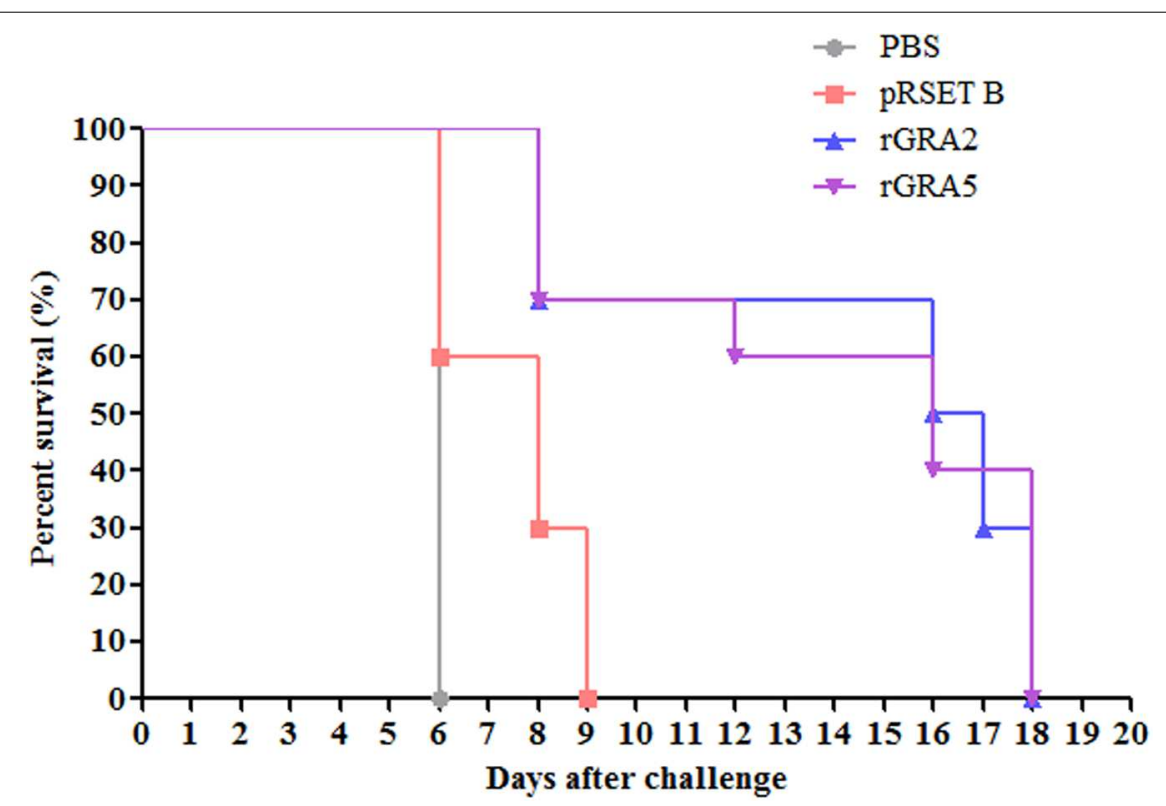

FIGURE 7 | Survival rate of the immunized mice. All four groups of the immunized mice (PBS, pRSET B, rGRA2, rGRA5) were subjected to lethal challenge with 1000 live tachyzoites of $T$. gondii virulent RH strain 2 weeks after the last immunization. Mice immunized with rGRA2 and rGRA5 exhibited a significant increase in the survival days (median survival of 16.5 and 16 days respectively) in comparison to the control mice injected with PBS and pRSET B (median survival of 6 and 8 days respectively). Each group consisted of 10 mice.

was to enhance the immunogenicity of the subunit vaccine as well as directing the immune response toward Th1.

Immunogenicity and protective efficacy of several $T$. gondii recombinant antigens produced in bacteria have also been performed against Toxoplasma infection in experimental mouse models (Martin et al., 2004; Dziadek et al., 2009, 2012). It was reported that alum adjuvant-formulated rGRA4 was a potential multi-antigen vaccination candidate against chronic T. gondii infection either alone or in combination with rROP2 which were both produced in $\mathrm{pQE}$ expression vector. It protected the vaccinated $\mathrm{C} 57 \mathrm{BL} / 6$ and $\mathrm{C} 3 \mathrm{H}$ mice against challenge with ME49 strain through brain cyst reduction (Martin et al., 2004).
Meanwhile, vaccination of $\mathrm{C} 3 \mathrm{H} / \mathrm{HeJ}$ mice with $\mathrm{rROP} 2$ and rROP4 which were expressed in $\mathrm{pH}$ is vector has been shown to elicit mixed Th1/Th2-type immune response with specific IL2 production. These two antigens conferred partial protection against challenge with DX strain (low virulent) with $46 \%$ brain cysts reduction. It was also reported that combination of the same rROP2 and rROP4 antigens with either rGRA4 or rSAG1 triggered both humoral (generation of high levels of IgG1 and IgG2a) and cellular- (secretion of IFN- $\gamma$ and IL-2) associated immunity. The brain cysts loads in the vaccinated BALB/c mice were greatly decreased ( 84 and $77 \%$ reduction respectively) compared to PBS-injected mice (Dziadek et al., 2009, 2012). 
In this study, immunization of BALB/c mice with rGRA2 and rGRA5 emulsified with complete/incomplete Freund's adjuvant (C/IFA) successfully triggered both humoral and cellular mixed Th1/Th2-like immune responses, predominantly Th1 in the vaccinated mice. The triggered immune response eventually prolonged the mice's survival rates against lethal challenge with the virulent RH strain of $T$. gondii. These results confirmed the antigenicity and immunogenicity of the two recombinant proteins. However, Freund's adjuvant is not suitable for use in humans due to safety concerns (Stills, 2005).

Analysis of IgG antibody through SDS-PAGE/WB and ELISA indicated that anti-GRA2 and anti-GRA5 IgG antibody was produced in the immunized mice 2 weeks after prime injection. The antibody levels increased with successive immunization where antibody titres ranged from $1: 409,600$ to $1: 819200$ at 6 weeks post-prime injection. Meanwhile, anti-GRAs IgG antibody detection was not observed in Native-PAGE/WB probably due to protein folding which hinders the B-cell epitope. Besides, relatively high levels of IgG1 and IgG2a were detected in the immunized mice serum. The levels of these two antibody isotypes are almost similar with slightly higher IgG1 than that of IgG2a. Production of IgG1 is Th2 related, while IgG2a is associated with Th1-driven immunity (Germann et al., 1995).

$\mathrm{CD} 4^{+} \mathrm{Th} 1$ cell populations are involved in B cells activation and subclass-switched antibody production, whereby its absence would lead to increased susceptibility to $T$. gondii (Harris et al., 2001; Johnson and Sayles, 2002). Induction of humoral immune response plays an essential role in the resistance against Toxoplasma infection in which most of the B celldeficient mice survived from the infection post-treatment with immune serum (Frenkel and Taylor, 1982). Significant protection elicited through intraperitoneal injection of monoclonal antiT. gondii surface antigen antibody into mice against moderately and highly virulent Toxoplasma infection further supports the importance of humoral immunity in fighting toxoplasmosis (Johnson et al., 1983). Other than conferring resistance to T. gondii acute infection and controlling its chronic infection, humoral immunity has been demonstrated to be important in protecting rodents against other protozoa parasites as well, such as Plasmodium berghei yoelii (Weinbaum et al., 1976) and Trypanosoma cruzi (Rodriguez et al., 1981).

A study on the protective and resisting roles of B cells response against lethal challenge with virulent strains of $T$. gondii demonstrated that antigen-specific antibody inhibited host cell active invasion by blocking the tachyzoites directly, preventing them from attaching to the host cell and thus restricting parasite propagation (Sayles et al., 2000). On the other hand, antibody-coated tachyzoites could be destroyed by phagocytic cells such as macrophage through passive phagocytosis (Sibley et al., 1993). Positive correlation was observed between the high titers of two IgG isotypes; IgG1 and IgG2a and the levels of phagocytosis which eventually protected immunized mice against S. pneumonia (Lefeber et al., 2003).

Earlier findings reported that monoclonal antibody against T. gondii surface antigens successfully blocked tachyzoites invasion and in vitro propagation as compared to monoclonal antibody against antigens of $T$. gondii secretory organelles with little or no effect on invasion (Johnson et al., 1983; Grimwood and Smith, 1996). However, another study showed that phagocytic cell (macrophage) invasion of $T$. gondii was inhibited by monoclonal anti-GRA2 in the presence of complement (Cha et al., 2001). At the same time, the monoclonal antibody partially protected mice against RH strain tachyzoite infection mediated by complement-dependent effector mechanism (Sayles et al., 2000; Cha et al., 2001). This highlights the protective role played by specific antibody.

Apart from humoral immunity, cell-mediated immunity is a major protective response against intracellular $T$. gondii. The cellmediated immunity response is through specific $\mathrm{T}$ lymphocytes activation $\left(\mathrm{CD}^{+}\right.$and $\left.\mathrm{CD}^{+}\right)$, especially Th1 response coupled with IFN- $\gamma$ production (Suzuki and Remington, 1988; Gazzinelli et al., 1991; Parker et al., 1991; Sibley et al., 1993). Interferongamma-mediated cytotoxic $\mathrm{T}$ lymphocyte (CTL) response restraints the propagation and spreading of the parasite by impeding the growth of actively dividing tachyzoites (acute phase) and limiting reactivation of the encysted bradyzoites (Pfefferkorn, 1984; Pfefferkorn et al., 1986; Suzuki et al., 1988; Dillon et al., 1992; Zheng et al., 2013).

In this study, stimulated T-lymphocytes in the spleen cells of rGRA2- and rGRA5-immunized mice proliferated significantly. Interferon-gamma and IL-2 were two pro-inflammatory cytokines that were secreted in large amount suggesting that $\mathrm{CD}^{+}$Th1 and $\mathrm{CD}^{+}$cytotoxic T-cells were being triggered. In addition, IL-4 and IL-10 were also produced but at relatively low levels which are associated with $\mathrm{CD} 4^{+} \mathrm{Th} 2$ cells induction. Besides fighting against $T$. gondii, these two anti-inflammatory cytokines play a vital role in balancing and reducing the deleterious inflammatory effect, especially of IFN- $\gamma$ (Snapper and Paul, 1987; Gazzinelli et al., 1991; Bessieres et al., 1997).

Humoral and cellular immune responses are interrelated and synergistic instead of acting alone to mount protection against any pathogen. Activated cytokine-secreting Th cells are involved in the stimulation of antibody-producing B cells as well as determining the switch of antibody isotype, either IgG1 or IgG2a in T-cell dependent immunity (Snapper and Paul, 1987; Germann et al., 1995). Th1-related IFN- $\gamma$ induces IgG2a generation and expression of the respective FcR1 on mouse macrophage (equivalent to human monocyte $\mathrm{FcR}$ ), suppressing IgG1 synthesis at the same time. In other words, Th1 is responsible for macrophage activation through stimulation of pro-inflammatory cytokines (IFN- $\gamma$, TNF- $\alpha$, IL-2) and IgG2a antibody generation. The protective role of IFN- $\gamma$ and IgG2a has been demonstrated through opsonisation, complement-mediated cell lysis and antibody-dependent cellular cytotoxicity (ADCC) (Johnson et al., 1985; Snapper and Paul, 1987; Petersen et al., 1998). On the other hand, Th2-related IL-4, which is also known as B cell stimulatory factor-1 (BSF-1), possesses an antagonistic effect to IFN- $\gamma$ whereby it enhances IgG1 production and FcR2 expression on mouse macrophage (equivalent to natural killer cell FcR in human) but suppresses IgG2a production (Perussia et al., 1983; Snapper and Paul, 1987). Th2 stimulates development of anti-inflammatory cytokines (IL-4, IL-5, IL-6, and IL-10) and IgG1 antibody which leads to down regulation of macrophage activity (Petersen et al., 1998). 
A study demonstrated that sterile protective immunity to toxoplasmosis is possible if strong humoral and cellular immune responses are successfully elicited and acting synergistically whereby the survival rates of the infected mice will not significantly increase in the absence of either antibody production or T cell immunity (Frenkel and Taylor, 1982). Therefore, it is deduced that the increase in the median survival time of T. gondii-challenged mice from 6-8 days (non-immunized mice; PBS and pRSET B-injected) to 16-16.5 days (immunized mice; rGRA5 and rGRA2-injected) in this study might be resulted from the interaction between antibody-producing B lymphocytes and cytokine-producing T lymphocytes (Th1 and Th2).

The delayed onset of death observed in rGRA2 and rGRA5immunized mice might be due to the interplay between Th1 and Th2-driven responses as findings have shown that Th1related cytokine primarily IFN- $\gamma$ causes early mortality whereas Th2-related IL-4 and IL-10 diminish short-term fatality by down regulating Th1 response and thus reducing the severe inflammatory effect provoked by IFN- $\gamma$ at the early acute phase of toxoplasmosis (Roberts et al., 1996; Neyer et al., 1997; Petersen et al., 1998). An increase in survival rate and decrease in necrosis of the small intestine was observed in C57BL/6 mice treated with monoclonal anti-IFN- $\gamma$ antibody (Liesenfeld et al., 1996). It has also been determined that secretion of IFN- $\gamma$ is directly proportional to the mortality rate of the infected mice (McLeod et al., 1989). IL-4-deficient mice have higher susceptibility toward acute Toxoplasma infection compared to the wild type due to excessive IFN- $\gamma$ secretion. In contrast, development of necrotic lesions with free living tachyzoites has been seen in wild-type mice but not in IL-4-deficient mice (Roberts et al., 1996). One of the negative regulatory effects of IL-10 is to suppress macrophage killing activity mediated by IFN- $\gamma$ (Sibley et al., 1993). Infected IL-10-depleted mice died during acute Toxoplasma infection with relatively high levels of IFN- $\gamma$ and IL-12 detected in their serum. These mice were believed to have succumbed to lethal immunopathology instead of parasitic infection as there was no sign of significant T. gondii propagation (Gazzinelli et al., 1996).

The overall results obtained in the present study are in agreement with the results of previous studies. The recombinant antigens triggered strong humoral and cellular Th1-dominating immune response by up-regulating the development of antigenspecific IgG antibody (IgG2a) and Th1-related cytokines (IFN- $\gamma$ and IL-2) (Golkar et al., 2007; Zhou et al., 2007). Monophosphoryl lipid A (MPL) adjuvant-formulated rGRA2 had been shown to reduce brain cysts formation significantly in

\section{REFERENCES}

Bessieres, M. H., Swierczynski, B., Cassaing, S., Miedouge, M., Olle, P., Seguela, J. P., et al. (1997). Role of IFN-gamma, TNF-alpha, IL4 and IL10 in the regulation of experimental Toxoplasma gondii infection. J. Eukaryot. Microbiol. 44, 87S. doi: 10.1111/j.1550-7408.1997.tb05800.x

Bhopale, G. M. (2003). Development of a vaccine for toxoplasmosis: current status. Microbes Infect. 5, 457-462. doi: 10.1016/S1286-4579(03)00048-0

Bruna-Romero, O., Oliveira, D. M., and Andrade-Neto, V. F. (2012). "Toxoplasmosis: advances and vaccine perspectives," in Current Topics in Tropical Medicine, ed. A. Rodriguesz-Morales (Rijeka: InTech), 169-184. the immunized CBA/J mice either alone or mixed with rGRA6, thereby protecting against chronic T. gondii infection (Golkar et al., 2007). Immunization of BALB/c mice with multi-antigenic protein vaccine containing SAG1-GRA2 expressed in yeast host successfully increased survival time of the vaccinated mice up to 15 days against lethal challenge with $T$. gondii $\mathrm{RH}$ strain (acute infection) (Zhou et al., 2007).

Protective efficacy of GRA5 subunit vaccine against chronic toxoplasmosis has been indicated by intranasal immunization in combination with rGRA7 and rROP2 adjuvanted with cholera toxin by reducing brain cyst formation of VEG strain by $58.3 \%$ in BALB/c mice (Igarashi et al., 2008b). Nevertheless, this is thus far the first report of evaluation of the immunity elicited by GRA 5 as a single-antigenic subunit vaccine candidate against acute toxoplasmosis in the mouse model.

\section{CONCLUSION}

Subcutaneous injection of mice with subunit vaccines rGRA2 and rGRA5 successfully triggered humoral and cellular responses which resulted in partial protection to the vaccinated mice against parasitic lethal challenge. A combination of Th1/Th2-related responses primarily Th1 was obtained with significant increased production of IgG2a, IFN- $\gamma$, IL- 2 and IgG1 but relatively low level of IL-4 and IL-10. The encouraging findings obtained in this study provide a basis for further investigation into the development of a recombinant multi-antigenic candidate using combination of GRA2-GRA5 for immunization against T. gondii infection.

\section{AUTHOR CONTRIBUTIONS}

YL and MY conceived and designed the study, and critically revised the manuscript. XT performed the experiments, analyzed the data and drafted the manuscript. All authors read and approved the final manuscript.

\section{ACKNOWLEDGMENTS}

This research project was supported by University of Malaya High Impact Research (HIR) Grant UM-MOHE (UM.C/HIR/MOHE/MED/16) from the Ministry of Higher Education, Malaysia.

Buxton, D. (1998). Protozoan infections (Toxoplasma gondii, Neospora caninum and Sarcocystis spp.) in sheep and goats: recent advances. Vet Res. 29, 289-310.

Capron, A., and Dessaint, J. P. (1988). Vaccination against parasitic diseases: some alternative concepts for the definition of protective antigens. Ann. Inst. Pasteur Immunol. 139, 109-117. doi: 10.1016/0769-2625(88) 90135-3

Cesbron-Delauw, M. F., and Capron, A. (1993). Excreted/secreted antigens of Toxoplasma gondii-their origin and role in the host-parasite interaction. Res. Immunol. 144, 41-44. doi: 10.1016/S0923-2494(05)80096-3

Cha, D. Y., Song, I. K., Lee, G. S., Hwang, O. S., Noh, H. J., Yeo, S. D., et al. (2001). Effects of specific monoclonal antibodies to dense granular proteins on 
the invasion of Toxoplasma gondii in vitro and in vivo. Korean J. Parasitol. 39, 233-240. doi: 10.3347/kjp.2001.39.3.233

Chen, R., Lu, S. H., Tong, Q. B., Lou, D., Shi, D. Y., Jia, B. B., et al. (2009). Protective effect of DNA-mediated immunization with liposome-encapsulated GRA4 against infection of Toxoplasma gondii. J. Zhejiang Univ. Sci. B 10, 512-521. doi: 10.1631/jzus.B0820300

Ching, X. T., Lau, Y. L., and Fong, M. Y. (2015). Heterologous expression of Toxoplasma gondii dense granule protein 2 and 5. Southeast Asian J. Trop. Med. Public Health 46, 375-387.

Denkers, E. Y., and Gazzinelli, R. T. (1998). Regulation and function of T-cellmediated immunity during Toxoplasma gondii infection. Clin. Microbiol. Rev. $11,569-588$

Dillon, S. B., Demuth, S. G., Schneider, M. A., Weston, C. B., Jones, C. S., Young, J. F., et al. (1992). Induction of protective class I MHC-restricted CTL in mice by a recombinant influenza vaccine in aluminium hydroxide adjuvant. Vaccine 10, 309-318. doi: 10.1016/0264-410X(92)90369-U

Dubey, J. P. (2010). Toxoplasmosis of Animals and Humans, 2nd Edn. Boca Raton, FL: CRC Press, 313.

Dziadek, B., Gatkowska, J., Brzostek, A., Dziadek, J., Dzitko, K., and Dlugonska, H. (2009). Toxoplasma gondii: the immunogenic and protective efficacy of recombinant ROP2 and ROP4 rhoptry proteins in murine experimental toxoplasmosis. Exp. Parasitol. 123, 81-89. doi: 10.1016/j.exppara.2009.06.002

Dziadek, B., Gatkowska, J., Grzybowski, M., Dziadek, J., Dzitko, K., and Dlugonska, H. (2012). Toxoplasma gondii: the vaccine potential of three trivalent antigen-cocktails composed of recombinant ROP2, ROP4, GRA4 and SAG1 proteins against chronic toxoplasmosis in BALB/c mice. Exp. Parasitol. 131, 133-138. doi: 10.1016/j.exppara.2012.02.026

Echeverria, P. C., De Miguel, N., Costas, M., and Angel, S. O. (2006). Potent antigen-specific immunity to Toxoplasma gondii in adjuvant-free vaccination system using Rop2-Leishmania infantum Hsp83 fusion protein. Vaccine 24, 4102-4110. doi: 10.1016/j.vaccine.2006.02.039

Feng, P., Park, J., Lee, B. S., Lee, S. H., Bram, R. J., and Jung, J. U. (2002). Kaposi's sarcoma-associated herpesvirus mitochondrial K7 protein targets a cellular calcium-modulating cyclophilin ligand to modulate intracellular calcium concentration and inhibit apoptosis. J. Virol. 76, 11491-11504. doi: 10.1128/JVI.76.22.11491-11504.2002

Frenkel, J. K., and Taylor, D. W. (1982). Toxoplasmosis in immunoglobulin M-suppressed mice. Infect. Immun. 38, 360-367.

Gazzinelli, R. T., Hakim, F. T., Hieny, S., Shearer, G. M., and Sher, A. (1991). Synergistic role of $\mathrm{CD} 4+$ and $\mathrm{CD} 8+\mathrm{T}$ lymphocytes in IFN-gamma production and protective immunity induced by an attenuated Toxoplasma gondii vaccine. J. Immunol. 146, 286-292.

Gazzinelli, R. T., Wysocka, M., Hieny, S., Scharton-Kersten, T., Cheever, A., Kuhn, R., et al. (1996). In the absence of endogenous IL-10, mice acutely infected with Toxoplasma gondii succumb to a lethal immune response dependent on CD4+ T cells and accompanied by overproduction of IL-12, IFN-gamma and TNF-alpha. J. Immunol. 157, 798-805.

Germann, T., Bongartz, M., Dlugonska, H., Hess, H., Schmitt, E., Kolbe, L., et al. (1995). Interleukin-12 profoundly up-regulates the synthesis of antigen-specific complement-fixing IgG2a, IgG2b and IgG3 antibody subclasses in vivo. Eur. J. Immunol. 25, 823-829. doi: 10.1002/eji.1830250329

Golkar, M., Shokrgozar, M. A., Rafati, S., Musset, K., Assmar, M., Sadaie, R., et al. (2007). Evaluation of protective effect of recombinant dense granule antigens GRA2 and GRA6 formulated in monophosphoryl lipid A (MPL) adjuvant against Toxoplasma chronic infection in mice. Vaccine 25, 4301-4311. doi: 10.1016/j.vaccine.2007.02.057

Grimwood, J., and Smith, J. E. (1996). Toxoplasma gondii: the role of parasite surface and secreted proteins in host cell invasion. Int. J. Parasitol. 26, 169-173. doi: 10.1016/0020-7519(95)00103-4

Harris, D. P., Koch, S., Mullen, L. M., and Swain, S. L. (2001). B cell immunodeficiency fails to develop in CD4-deficient mice infected with BM5: murine AIDS as a multistep disease. J. Immunol. 166, 6041-6049. doi: 10.4049/jimmunol.166.10.6041

Hill, D., and Dubey, J. P. (2002). Toxoplasma gondii: transmission, diagnosis and prevention. Clin. Microbiol. Infect. 8, 634-640. doi: 10.1046/j.14690691.2002.00485.x

Hiszczynska-Sawicka, E., Oledzka, G., Holec-Gasior, L., Li, H., Xu, J. B., Sedcole, R., et al. (2011). Evaluation of immune responses in sheep induced by DNA immunization with genes encoding GRA1, GRA4, GRA6 and GRA7 antigens of Toxoplasma gondii. Vet. Parasitol. 177, 281-289. doi: 10.1016/j.vetpar.2010.11.047

Igarashi, M., Kano, F., Tamekuni, K., Kawasaki, P. M., Navarro, I. T., Vidotto, O., et al. (2008a). Toxoplasma gondii: cloning, sequencing, expression, and antigenic characterization of ROP2, GRA5 and GRA7. Genet. Mol. Res. 7, 305-313. doi: 10.4238/vol7-2gmr423

Igarashi, M., Kano, F., Tamekuni, K., Machado, R. Z., Navarro, I. T., Vidotto, O., et al. (2008b). Toxoplasma gondii: evaluation of an intranasal vaccine using recombinant proteins against brain cyst formation in BALB/c mice. Exp. Parasitol. 118, 386-392. doi: 10.1016/j.exppara.2007.10.002

Innes, E. A. (2010). Vaccination against Toxoplasma gondii: an increasing priority for collaborative research? Expert Rev. Vaccines 9, 1117-1119. doi: 10.1586/erv.10.113

Ismael, A. B., Sekkai, D., Collin, C., Bout, D., and Mevelec, M. N. (2003). The MIC3 gene of Toxoplasma gondii is a novel potent vaccine candidate against toxoplasmosis. Infect. Immun. 71, 6222-6228. doi: 10.1128/IAI.71.11.62226228.2003

Jackson, M. H., and Hutchison, W. M. (1989). The prevalence and source of Toxoplasma infection in the environment. Adv. Parasitol. 28, 55-105. doi: 10.1016/S0065-308X(08)60331-0

Johnson, A. M., Mcdonald, P. J., and Neoh, S. H. (1983). Monoclonal antibodies to Toxoplasma cell membrane surface antigens protect mice from toxoplasmosis. J. Protozool. 30, 351-356. doi: 10.1111/j.1550-7408.1983.tb02929.x

Johnson, L. L., and Sayles, P. C. (2002). Deficient humoral responses underlie susceptibility to Toxoplasma gondii in CD4-deficient mice. Infect. Immun. 70, 185-191. doi: 10.1128/IAI.70.1.185-191.2002

Johnson, W. J., Steplewski, Z., Koprowski, H., and Adams, D. O. (1985). "Destructive interactions between murine macrophages, tumor cells, and antibodies of the IgG2a isotype," in Mechanisms of Cell-Mediated Cytotoxicity II, eds P. Henkart and E. Martz (Berlin: Springer), 75-80.

Kaplan, E. L., and Meier, P. (1958). Nonparametric estimation from incomplete observations. J. Am. Stat. Assoc. 53, 457-481. doi: 10.1080/01621459.1958.10501452

Kur, J., Holec-Gasior, L., and Hiszczynska-Sawicka, E. (2009). Current status of toxoplasmosis vaccine development. Expert Rev. Vaccines 8, 791-808. doi: 10.1586/erv.09.27

Lefeber, D. J., Benaissa-Trouw, B., Vliegenthart, J. F., Kamerling, J. P., Jansen, W. T., Kraaijeveld, K., et al. (2003). Th1-directing adjuvants increase the immunogenicity of oligosaccharide-protein conjugate vaccines related to Streptococcus pneumoniae type 3. Infect. Immun. 71, 6915-6920. doi: 10.1128/IAI.71.12.6915-6920.2003

Liesenfeld, O., Kosek, J., Remington, J. S., and Suzuki, Y. (1996). Association of CD4+ T cell-dependent, interferon-gamma-mediated necrosis of the small intestine with genetic susceptibility of mice to peroral infection with Toxoplasma gondii. J. Exp. Med. 184, 597-607. doi: 10.1084/jem.184.2.597

Liu, S., Shi, L., Cheng, Y. B., Fan, G. X., Ren, H. X., and Yuan, Y. K. (2009). Evaluation of protective effect of multi-epitope DNA vaccine encoding six antigen segments of Toxoplasma gondii in mice. Parasitol. Res. 105, 267-274. doi: $10.1007 /$ s00436-009-1393-1

Martin, V., Supanitsky, A., Echeverria, P. C., Litwin, S., Tanos, T., De Roodt, A. R., et al. (2004). Recombinant GRA4 or ROP2 protein combined with alum or the gra4 gene provides partial protection in chronic murine models of toxoplasmosis. Clin. Diagn. Lab. Immunol. 11, 704-710.

McLeod, R., Eisenhauer, P., Mack, D., Brown, C., Filice, G., and Spitalny, G. (1989). Immune responses associated with early survival after peroral infection with Toxoplasma gondii. J. Immunol. 142, 3247-3255.

Montoya, J. G., and Liesenfeld, O. (2004). Toxoplasmosis. Lancet 363, 1965-1976. doi: 10.1016/S0140-6736(04)16412-X

Nam, H. W. (2009). GRA proteins of Toxoplasma gondii: maintenance of hostparasite interactions across the parasitophorous vacuolar membrane. Korean J. Parasitol. 47(Suppl.), S29-S37. doi: 10.3347/kjp.2009.47.S.S29

Neyer, L. E., Grunig, G., Fort, M., Remington, J. S., Rennick, D., and Hunter, C. A. (1997). Role of interleukin-10 in regulation of T-cell-dependent and T-cellindependent mechanisms of resistance to Toxoplasma gondii. Infect. Immun. $65,1675-1682$.

Parker, S. J., Roberts, C. W., and Alexander, J. (1991). CD8+ T cells are the major lymphocyte subpopulation involved in the protective immune 
response to Toxoplasma gondii in mice. Clin. Exp. Immunol. 84, 207-212. doi: 10.1111/j.1365-2249.1991.tb08150.x

Perussia, B., Dayton, E. T., Lazarus, R., Fanning, V., and Trinchieri, G. (1983). Immune interferon induces the receptor for monomeric IgG1 on human monocytic and myeloid cells. J. Exp. Med. 158, 1092-1113. doi: 10.1084/jem.158.4.1092

Petersen, E., Nielsen, H. V., Christiansen, L., and Spenter, J. (1998). Immunization with $E$. coli produced recombinant $T$. gondii SAG1 with alum as adjuvant protect mice against lethal infection with Toxoplasma gondii. Vaccine 16, 1283-1289. doi: 10.1016/S0264-410X(98)00039-5

Pfefferkorn, E. R. (1984). Interferon-gamma blocks the growth of Toxoplasma gondii in human fibroblasts by inducing the host cells to degrade tryptophan. Proc. Natl. Acad. Sci. U.S.A. 81, 908-912. doi: 10.1073/pnas.81.3.908

Pfefferkorn, E. R., Rebhun, S., and Eckel, M. (1986). Characterization of an indoleamine 2,3-dioxygenase induced by gamma-interferon in cultured human fibroblasts. J. Interferon Res. 6, 267-279. doi: 10.1089/jir.1986.6.267

Roberts, C. W., Ferguson, D. J., Jebbari, H., Satoskar, A., Bluethmann, H., and Alexander, J. (1996). Different roles for interleukin-4 during the course of Toxoplasma gondii infection. Infect. Immun. 64, 897-904.

Rodriguez, A. M., Santoro, F., Afchain, D., Bazin, H., and Capron, A. (1981). Trypanosoma cruzi infection in B-cell-deficient rats. Infect. Immun. 31, 524529.

Sayles, P. C., Gibson, G. W., and Johnson, L. L. (2000). B cells are essential for vaccination-induced resistance to virulent Toxoplasma gondii. Infect. Immun. 68, 1026-1033. doi: 10.1128/IAI.68.3.1026-1033.2000

Schaap, D., Vermeulen, A. N., Roberts, C. W., and Alexander, J. (2007). "Vaccination against toxoplasmosis: current status and future prospects," in Toxoplasma gondii: The Model Apicomplexan. Perspectives and Methods, 1st Edn, eds L. M. Weiss and K. Kim (Cambridge, MA: Academic Press).

Scorza, T., D'souza, S., Laloup, M., Dewit, J., De Braekeleer, J., Verschueren, H., et al. (2003). A GRA1 DNA vaccine primes cytolytic CD8+ T cells to control acute Toxoplasma gondii infection. Infect. Immun. 71, 309-316. doi: 10.1128/IAI.71.1.309-316.2003

Sibley, L. D., Adams, L. B., and Krahenbuhl, J. L. (1993). Macrophage interactions in toxoplasmosis. Res. Immunol. 144, 38-40. doi: 10.1016/S09232494(05)80095-1

Sloat, B. R., Sandoval, M. A., Hau, A. M., He, Y., and Cui, Z. (2010). Strong antibody responses induced by protein antigens conjugated onto the surface of lecithin-based nanoparticles. J. Control. Release 141, 93-100. doi: 10.1016/j.jconrel.2009.08.023

Snapper, C. M., and Paul, W. E. (1987). Interferon-gamma and B cell stimulatory factor-1 reciprocally regulate Ig isotype production. Science 236, 944-947. doi: $10.1126 /$ science. 3107127

Stills, H. F. Jr. (2005). Adjuvants and antibody production: dispelling the myths associated with Freund's complete and other adjuvants. ILAR J. 46, 280-293. doi: 10.1093/ilar.46.3.280
Sun, X., Mei, M., Zhang, X., Han, F., Jia, B., Wei, X., et al. (2014). The extracellular matrix protein mindin as a novel adjuvant elicits stronger immune responses for rBAG1, rSRS4 and rSRS9 antigens of Toxoplasma gondii in BALB/c mice. BMC Infect. Dis. 14:429. doi: 10.1186/1471-233414-429

Sun, X. M., Zou, J., Aa, E. S., Yan, W. C., Liu, X. Y., Suo, X., et al. (2011). DNA vaccination with a gene encoding Toxoplasma gondii GRA6 induces partial protection against toxoplasmosis in BALB/c mice. Parasit. Vectors 4:213. doi: 10.1186/1756-3305-4-213

Suzuki, Y., Orellana, M. A., Schreiber, R. D., and Remington, J. S. (1988). Interferon-gamma: the major mediator of resistance against Toxoplasma gondii. Science 240, 516-518. doi: 10.1126/science.31 28869

Suzuki, Y., and Remington, J. S. (1988). Dual regulation of resistance against Toxoplasma gondii infection by Lyt-2+ and Lyt-1+, L3T4+ T cells in mice. J. Immunol. 140, 3943-3946.

Tilley, M., Fichera, M. E., Jerome, M. E., Roos, D. S., and White, M. W. (1997). Toxoplasma gondii sporozoites form a transient parasitophorous vacuole that is impermeable and contains only a subset of dense-granule proteins. Infect. Immun. 65, 4598-4605.

Weinbaum, F. I., Evans, C. B., and Tigelaar, R. E. (1976). Immunity to Plasmodium berghei yoelii in mice. I. The course of infection in $\mathrm{T}$ cell and $\mathrm{B}$ cell deficient mice. J. Immunol. 117, 1999-2005.

Xue, M., He, S., Cui, Y., Yao, Y., and Wang, H. (2008). Evaluation of the immune response elicited by multi-antigenic DNA vaccine expressing SAG1, ROP2 and GRA2 against Toxoplasma gondii. Parasitol. Int. 57, 424-429. doi: 10.1016/j.parint.2008.05.001

Zheng, B., Lu, S., Tong, Q., Kong, Q., and Lou, D. (2013). The virulencerelated rhoptry protein 5 (ROP5) of Toxoplasma Gondii is a novel vaccine candidate against toxoplasmosis in mice. Vaccine 31, 4578-4584. doi: 10.1016/j.vaccine.2013.07.058

Zhou, H., Gu, Q., Zhao, Q., Zhang, J., Cong, H., Li, Y., et al. (2007). Toxoplasma gondii: expression and characterization of a recombinant protein containing SAG1 and GRA2 in Pichia pastoris. Parasitol. Res. 100, 829-835. doi: 10.1007/s00436-006-0341-6

Conflict of Interest Statement: The authors declare that the research was conducted in the absence of any commercial or financial relationships that could be construed as a potential conflict of interest.

Copyright (c) 2016 Ching, Fong and Lau. This is an open-access article distributed under the terms of the Creative Commons Attribution License (CC BY). The use, distribution or reproduction in other forums is permitted, provided the original author(s) or licensor are credited and that the original publication in this journal is cited, in accordance with accepted academic practice. No use, distribution or reproduction is permitted which does not comply with these terms. 